\title{
The Relativistic Wave Equation
}

\author{
Ilija Barukčić
}

\begin{abstract}
In general, it is well known that the Schrödinger equation is not compatible with special relativity theory to a necessary extent. Thus far, there are already several trials to formulate versions of the Schrödinger equation to ensure compatibility with special relativity theory, the Klein-Gordon-equation or the Dirac equation are some of these attempts.

Material and Methods: In this paper, Einstein's relativistic energy momentum relation is re-analyzed, a normalized relativistic energy momentum relation is derived. The derived normalized relativistic energy momentum relation together with the known Schrödinger equation is used as a starting point to establish a wave equation consistent with special relativity theory.

Results: In this publication, based on Einstein's relativistic energy momentum relation, the historical problem of the "particle-wave-duality" is solved. Furthermore, a special relativity theory consistent wave equation is derived.
\end{abstract}

Index Terms-Causality, quantum mechanics, special and general relativity, unified field theory.

\section{INTRODUCTION}

The famous, long lasting dispute between Leibniz (conservation of vis viva or "kinetic" energy) and Newton (conservation of momentum) had its roots in a dispute between Gottfried Wilhelm Leibniz [1] (1646-1716) and followers of René Descartes (1596-1650) concerning Descartes' law of the quantity of motion, as discussed by Descartes in his Principia philosophiae of 1644. In fact, the core of this controversy was the dispute about the conservation of $\left[m_{r} \times v \times v\right]$ (Leibniz) versus the conservation of $\left[m_{r} \times v\right]$ (Newton [2]) through changes. According to Roger Boscovich (1745) and Jean d'Alembert (1758) both concepts are equally valid. Thus far, even today, it is a point of dispute, what is preserved through changes.

Under certain circumstances, the momentum $\left[m_{r} \times v\right]$ is part of Schrödinger's equation too. The Schrödinger's equation is still of importance especially for quantum mechanics. But it was Erwin Schrödinger himself who recognized that his equation is not relativity consistent and valid only under non-relativistic circumstances. In contrast to this, the principle of relativity, which is the foundation of the theory of special relativity, should be valid in quantum mechanics too. In other words, can the principles of quantum mechanics be reconciled with the principles of special relativity?

The Klein-Gordon-equation established by Oskar Klein and shortly afterwards deduced by Walter Gordon is one of these trials to reconcile relativity and quantum theory, the Dirac equation formulated by Dirac (1902-1984) another one.

Manuscript received June 2, 2013; revised December 23, 2013.

Ilija Barukčić is with Horandstrasse, Jever, Germany (e-mail: Barukcic@t-online.de).
Meanwhile, there are several other wave equations (Weyl equation, Majorana equation, Breit equation, Proca equation, Rarita-Schwinger equation, Bargmann-Wigner equations et cetera), each of them with different strength and reliability. But besides of all, a generally valid and invariant form of a relativity consistent wave equation or a fully self-consistent relativistic quantum theory is still not in sight.

\section{MATERIAL AND METHODS}

\section{A. Einstein's Mass-Energy Equivalence Relation}

According to Albert Einstein [3], it is

$$
m_{0}=m_{r} \times \sqrt[2]{1-\frac{v^{2}}{c^{2}}}
$$

or equally

$$
m_{0} \times c^{2}=m_{r} \times c^{2} \times \sqrt[2]{1-\frac{v^{2}}{c^{2}}}
$$

where $m_{0}$ denotes the "rest" mass, $m_{r}$ denotes the "relativistic" mass, $v$ denotes the relative velocity and $c$ denotes the speed of light in vacuum.

\section{B. The (Time-Dependent) Schrödinger Equation}

The Schrödinger equation [4] for any system, no matter whether relativistic or not, has the form

$$
\left(H-i \hbar \frac{\partial}{\partial t}\right) \Psi(t)=0
$$

where $H$ is the Hamiltonian and $\Psi(t)$ the wave function of the quantum system. The form of the Schrödinger equation itself depends on the physical situation.

\section{The Energy Operator}

In quantum mechanics, the Hamiltonian is a quantum mechanical operator corresponding to the total energy of a quantum mechanical system and usually denoted by $H$.

By analogy with classical mechanics, the Hamiltonian is the sum of operators corresponding to the potential and kinetic energies (of all the particles associated with a system) of a quantum mechanical system and can take different forms depending on the situation. The total energy operator is determined as

$$
H=i \hbar \frac{\partial}{\partial t}
$$

For our purposes, the (non-relativistic or relativistic) Hamiltonian is corresponding to the total energy of a quantum mechanical object. Thus far, using a suitable Hamiltonian operator (i.e. the problem of incorporating spin) 
is of fundamental importance.

\section{RESULtS}

A fundamental insight of special relativity is the relativistic energy-momentum relation. Even particles which propagate at velocities comparable to the speed of light $\mathrm{c}$ will obey this natural law. Thus far, extracting some mathematical and physical consequences from the relativistic energy-momentum relation is of use while establishing a generally valid relativistic wave equation. But a logically consistent relativistic description of quantum mechanical systems will not be achieved only by inserting the energy operator and momentum operator into the relativistic energy-momentum relation.

\section{A. The Normalized Relativistic Energy Momentum Relation}

Thus far, let $m_{0}$ denote the "rest mass", let $E_{0}=m_{0} \times c^{2}$ denote "the rest energy", let $m_{r}$ denote the "relativistic mass". In this context, let $E_{r}=m_{r} \times c^{2}$ denote "the relativistic energy", let $v$ denote the relative velocity, let $c$ denote the speed of light in vacuum. Further, let $p_{r}=m_{r} \times v$ denote the "relativistic momentum". In the following, let $E_{\text {Wave }}=p_{r} \times c$ denote the energy of the electromagnetic wave.

\section{Claim.}

In general, it is

$$
\frac{E_{0}^{2}}{E_{r}^{2}}+\frac{E_{\text {Wave }}^{2}}{E_{r}^{2}}=1
$$

\section{Proof.}

Starting with

$$
+1=+1
$$

it is equally

$$
1 \times m_{0}=1 \times m_{0}
$$

Based on Einstein's mass-energy equivalence (1) it follows that

$$
m_{0}=m_{r} \times \sqrt[2]{1-\frac{v^{2}}{c^{2}}}
$$

Rearranging this equation, we obtain

$$
\frac{m_{0}}{m_{r}}=\sqrt[2]{1-\frac{v^{2}}{c^{2}}}
$$

Let us perform a square operation of this equation. The result is

$$
\frac{m_{0}^{2}}{m_{r}^{2}}=1-\frac{v^{2}}{c^{2}}
$$

or the normalized relativistic energy momentum relation, a probability theory consistent formulation of the particle-wave duality, as

$$
\frac{m_{0}^{2}}{m_{r}^{2}}+\frac{v^{2}}{c^{2}}=1
$$

Rearranging the equation above yields

$$
\frac{m_{0}^{2} \times c^{4}}{m_{r}^{2} \times c^{4}}+\frac{v^{2} \times m_{r} \times m_{r} \times c^{2}}{c^{2} \times m_{r} \times m_{r} \times c^{2}}=1
$$

In general, the "rest energy" is $E_{0}^{2}=m_{0}^{2} \times c^{2} \times c^{2}$ while the "relativistic energy" is $E_{r}^{2}=m_{r}^{2} \times c^{2} \times c^{2}$ and the "relativistic momentum" is $p_{r}^{2}=v^{2} \times m_{r}^{2}$. Thus far, the equation before can be simplified as

$$
\frac{E_{0}^{2}}{E_{r}^{2}}+\frac{p_{\mathrm{r}}^{2} \times c^{2}}{E_{r}^{2}}=1
$$

The energy of the electromagnetic wave $E_{\text {Wave }}$ is known to be determined as $E_{\text {Wave }}=p_{r} \times c$. Based on this insight, the general form of the normalized relativistic energy momentum relation follows as

$$
\frac{E_{0}^{2}}{E_{r}^{2}}+\frac{E_{\mathrm{Wave}}^{2}}{E_{r}^{2}}=1
$$

\section{Q. e. d.}

A fundamental property of objective reality and thus far one of the most important features of quantum theory is that quantum mechanical objects or matter as such can exhibit both, wave and particle properties, simultaneously. Under different circumstances, either the particle aspects or the wave aspects of a quantum mechanical object may manifest themselves more strongly.

The simultaneous observation of wave and particle properties of quantum mechanical objects may be difficult as such. The question whether is it possible at all to measure simultaneously (collapse of the wave function) the extent, to what a quantum mechanical object has to be regarded as a particle or as a wave, is not the topic of this publication. These questions have long been debated and continue to be debated. Yet and besides of all, any single quantum mechanical object is determined by (14) too.

Thus far, in order to consider the contradictory nature of the particle-wave duality of quantum mechanical objects in greater detail, equation (14) show us to what extent something must be regarded as a wave and to what extent the same something must be regarded as being a particle. According to (14), the more a quantum mechanical object is a "particle" the less it is a "wave" and vice versa. It is worthwhile to state here that the one (i.e. the extent to which a quantum mechanical object is a particle) can be calculated, as soon as its own other (i.e. the extent to which a quantum mechanical object is a wave) is measured.

The historic problem of the particle-wave duality, deeply embedded into the foundations of quantum mechanics, is solved by (14).

\section{B. The Relativistic Wave Equation}

In general, however, just as proofed before, a "classical" or relativity based concept of "particle" and "waves" fully 
describes the behavior of quantum-scale objects too.

To put it slightly differently, nearly all physicists and philosophers will agree that the total energy of a physical system $\mathrm{S}$ cannot be different in quantum theory and in special relativity theory.

\section{1) Definitions}

Thus far, in what follows, let us consider the following. Let $m_{0}$ denote the "rest mass", let $E_{0}$ denote "the rest energy", let $m_{r}$ denote the "relativistic" mass, let $E_{\text {Wave }}$ denote the energy of the electromagnetic wave, let $E_{r}$ denote "the relativistic energy", let $v$ denote the relative velocity, let $c$ denote the speed of light in vacuum, let $E_{k}$ denote the relativistic kinetic energy, let $m_{p}$ denote the "relativistic potential" mass, let $E_{p}$ denote the relativistic potential energy, let $H$ denote the Hamiltonian, let $\Psi(t)$ denote the wave function, let $i$ denote the imaginary unit and let $h$ denote the reduced Planck constant. Let us define the relativistic kinetic energy $E_{k}$ as

$$
\begin{aligned}
E_{k}= & \frac{E_{\text {Wave }}{ }^{2}}{E_{r}} \\
= & m_{r} \times v^{2} \\
= & 2 \times\left(\frac{1}{2} \times m_{r} \times v^{2}\right) \\
= & \frac{m_{0}}{\sqrt{1-\frac{v^{2}}{c^{2}}} \times v^{2}} \\
= & m_{0} \times \frac{v^{2}}{\sqrt[2]{1-\frac{v^{2}}{c^{2}}}} \\
& =m_{0} \times \sqrt[2]{1-\frac{v^{2}}{c^{2}} \times c^{2}} \\
E_{p}= & \frac{E_{0}{ }^{2}}{E_{r}} \\
= & \frac{m_{0} \times c^{2} \times m_{0} \times c_{0}^{2} \times c^{2}}{m_{r} \times c^{2}} \\
& \frac{2}{1-\frac{v^{2}}{c^{2}}} \times c^{2} \\
&
\end{aligned}
$$

Let $\hat{E_{k}}$ denote the quantum mechanical operator of the relativistic kinetic energy. The quantum mechanical operator of relativistic kinetic energy is related to the historical concept of vis viva [5] and originally proposed by Gottfried Wilhelm Leibniz (1646-1716).

Further, let the relativistic potential energy $E_{p}$ be determined as

Let $\hat{E_{p}}$ denote the quantum mechanical operator of the relativistic potential energy. For our purposes it is important to note that the relativistic potential energy is the energy of a (high speed) particle (or of a system) due to the position of this (high speed) particle (or of this system).

The definition of the relativistic potential energy $E_{p}$ is backgrounded by Einstein's publication in 1907. According to Einstein [6], it is:

"Jeglicher Energie $E$ kommt also im Gravitationsfelde eine Energie der Lage zu, die ebenso groß ist, wie die Energie der Lage einer 'ponderablen' Masse von der Größe E/c²."

Translated into English:

"Thus, to each energy $E$ in the gravitational field there corresponds an energy of position that equals the potential energy of a "ponderable" mass of magnitude $\mathrm{E} / \mathrm{c}^{2}$."

\section{2) The relativistic wave equation}

\section{Claim.}

The total relativistic energy of a particle, $E_{r}$, is the sum of relativistic kinetic energy, $E_{k}$, and relativistic potential energy, $E_{p}$. Based on this assumption, the relativity theory consistent relativistic wave equation can be derived as

$$
\hat{E}_{p} \times \Psi(t)+\hat{E}_{k} \times \Psi(t)=H \times \Psi(t)=i \hbar \frac{\partial}{\partial t} \Psi(t)
$$

\section{Proof.}

Starting with

$$
+1=+1
$$

it is according to (10) at the end

$$
\frac{m_{0}^{2}}{m_{r}^{2}}+\frac{v^{2}}{c^{2}}=1
$$

Rearranging (19) yields

$$
\frac{m_{0}^{2} \times c^{2} \times c^{2}}{m_{r}^{2} \times c^{2} \times c^{2}}+\frac{m_{r} \times v^{2}}{m_{r} \times c^{2}}=1
$$

The relativistic kinetic energy $E_{k}$ was defined just before as $E_{k}=m_{r} \times v^{2}(15)$. Substituting this in (20), we obtain

$$
\frac{E_{0}^{2}}{E_{r}^{2}}+\frac{E_{k}}{E_{r}}=1
$$

The relativistic potential energy $E_{p}$ was defined above (16) as $E_{p}=E_{0}^{2} / E_{r}$ In other words, it is $E_{0}^{2}=E_{p} \times E_{r}(16)$. Based on (16) and (21) we obtain

$$
\frac{E_{p} \times E_{r}}{E_{r} \times E_{r}}+\frac{E_{k}}{E_{r}}=1
$$

Inside (22) $E_{r}$ cancel out. It follows that

$$
\frac{E_{p}}{E_{r}}+\frac{E_{k}}{E_{r}}=1
$$


Multiplying (23) by $E_{r}$ yields

$$
E_{p}+E_{k}=E_{r}
$$

The "total" relativistic energy $E_{r}$ is the sum of the relativistic potential $E_{p}$ and the relativistic kinetic energy $E_{k}$. Multiplying (23) by the Hamiltonian $\mathrm{H}$ and the wave function $\Psi(t)$ yields

$$
\frac{E_{p}}{E_{r}} \times H \times \Psi(t)+\frac{E_{k}}{E_{r}} \times H \times \Psi(t)=H \times \Psi(t)
$$

In special relativity theory, the total energy (of a quantum mechanical system) is corresponding to the "relativistic energy" $E_{r}$. Thus far, Klein-Gordon's [7], [8] and Dirac's [9] approach in mind, we equate

$$
E_{r}=H
$$

Rearranging (25), the (time-dependent) relativistic wave equation [for a single relativistic particle] ( $H$ and $E_{r}$ cancel out) follows as

$$
E_{p} \times \Psi(t)+E_{k} \times \Psi(t)=H \times \Psi(t)=i \hbar \frac{\partial}{\partial t} \Psi(t)
$$

The relativistic wave equation as derived above can be expressed in terms of quantum mechanical operators too. In terms of quantum mechanical operators, the special relativity consistent wave equation follows as

$$
\hat{E}_{p} \times \Psi(t)+\hat{E}_{k} \times \Psi(t)=H \times \Psi(t)=i \hbar \frac{\partial}{\partial t} \Psi(t)
$$

\section{Q.e.d.}

In plain language, the (time-dependent) relativistic wave equation means the total relativistic energy $E_{r}$ equals relativistic kinetic energy $E_{k}$ plus relativistic potential energy $E_{p}$. The following figure illustrates this relationship (24).

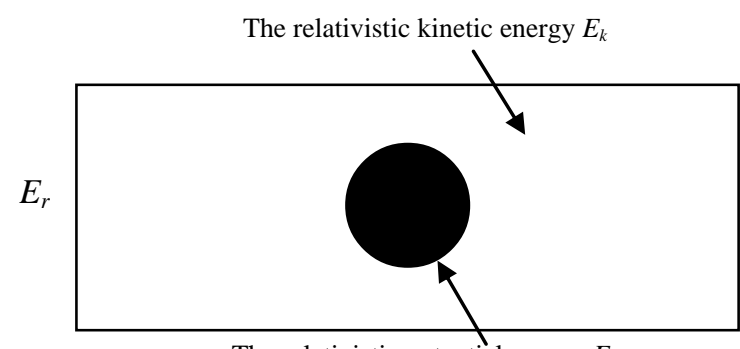

The relativistic potential energy $E$

Fig. 1. Illustration of the relativistic energy $E_{r}$.

\section{DISCUSSION}

In quantum mechanics, conditions, circumstances or the independently of human mind and consciousness existing objective reality is described by waves (the wave function) rather than with discrete particles. The establishment of quantum mechanical operators associated with the parameters needed to describe a quantum mechanical system in a relativity consistent way is of fundamental importance in this respect. Still, the quantum mechanical operator of the relativistic kinetic energy $\hat{E_{k}}$ and the quantum mechanical operator of the relativistic potential energy $\hat{E_{p}}$ have to be identified.

The quantum mechanical operator of the relativistic kinetic energy $\hat{E}_{k}$ can be constructed in the following way. Under some circumstances, the kinetic energy operator of quantum mechanics is known to be defined as

$$
\hat{T}=\frac{\hat{p_{x}{ }^{2}+\hat{p}_{y}^{2}+\hat{p}_{z}^{2}}}{2 \times m_{r}}
$$

Recall, it is

$$
\nabla^{2} \equiv \frac{\partial^{2}}{\partial x^{2}}+\frac{\partial^{2}}{\partial y^{2}}+\frac{\partial^{2}}{\partial z^{2}}
$$

Under these circumstances, the kinetic energy operator of quantum mechanics can be written more concisely as

$$
\hat{T}=-\frac{\hbar^{2}}{2 \times m_{r}} \nabla^{2}
$$

In general, the quantum mechanical operator of the relativistic kinetic energy is related to the kinetic energy operator of quantum mechanics in the following way. The formula of the kinetic energy in Newtonian $\left(E_{K N}\right)$ sense is known to be

$$
E_{K N}=\frac{1}{2} m_{r} \times v^{2}
$$

while the relativistic kinetic energy (15) is determined as

$$
E_{k}=2 \times\left(\frac{1}{2} m_{r} \times v=m_{r} \times v\right.
$$

Combining (31) and (32) together yields the quantum mechanical operator of the relativistic kinetic energy as

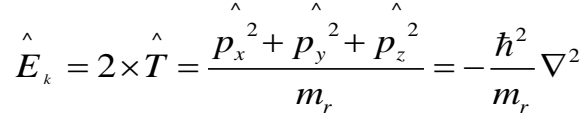

while the relative velocity squared can follow as

$$
v^{2}=\frac{\hat{p_{x}{ }^{2}+\hat{p}_{y}{ }^{2}+\hat{p}_{z}{ }^{2}}}{m_{r} \times m_{r}}=-\frac{\hbar^{2}}{m_{r} \times m_{r}} \nabla^{2}
$$

The finite ways of physics to manage the infinite are still a source of many difficulties in physics and in science as such. For our purposes it is important to note that with only a little further effort, the quantum mechanical operator of the relativistic potential energy $\hat{E_{p}}$ can be obtained in a similar way.

In our understanding, $m_{r}$ follows form the energy operator 
as

$$
m_{r}=\frac{i \hbar \frac{\partial}{\partial t}}{c^{2}}
$$

For our purposes, according to (16) and (27), the quantum mechanical operator of the relativistic potential energy $\hat{E_{p}}$ follows as

$$
\hat{E}_{p}=m_{0} \times m_{0} \times\left(\frac{c^{2} \times c^{2}}{i \hbar \frac{\partial}{\partial t}}\right)
$$

The relativistic wave equation in terms of quantum mechanical operators follows i.e. as

$$
\left(\frac{m_{0} \times m_{0} \times c^{2} \times c^{2}}{i \hbar \frac{\partial}{\partial t}}\right) \times \Psi(t)+\frac{\hat{p_{x}{ }^{2}+\hat{p}_{y}{ }^{2}+\hat{p}_{z}{ }^{2}}}{m_{r}} \times \Psi(t)=i \hbar \frac{\partial}{\partial t} \Psi(t)
$$

The square of the wave function $|\Psi|^{2}$ corresponds more or less to the necessity or to the probability of a particle to be at a given place at a given time. Further research is necessary to make the proof whether the relationship

$$
|\psi|^{2}=\frac{E_{p}}{E_{r}}=\frac{E_{0}^{2}}{E_{r}^{2}}=\frac{m_{0}^{2}}{m_{r}^{2}}
$$

or a similar one can be regarded as being correct.

\section{CONCLUSION}

Today the Copenhagen interpretation dominated quantum mechanics, a joint name for the ideas of Bohr, Heisenberg, Born and other physicists, is repeatedly regarded as the most successful scientific theory in the history of mankind. From a different but equivalent point of view, it is the same quantum theory which challenges not only our imagination but violates some fundamental principles (principle of causality) of modern science too.

Meanwhile, the historical dominance of the Copenhagen interpretation of quantum mechanics is no longer justified. In fact, the main pillars (principles) of today's Copenhagen interpretation dominated quantum mechanics like Heisenberg's uncertainty principle [10], Bell's theorem [11] and the CHSH inequality [12] are already refuted, but a fully, relativity consistent quantum theory is still not in sight.

This publication has solved the historical problem of the wave-particle duality. Based on Einstein's mass-energy equivalence and the normalized relativistic energy momentum relation, a relativity consistent wave equation is derived. The results of this publication can serve as the foundation of a relativity consistent quantum theory.

\section{REFERENCES}

[1] G. W. Leibniz, "Brevis demonstratio erroris memorabilis Cartesii et aliorum circa legem naturalem, secundum quam volunt a Deo eandem semper quantitatem motus conservari, qua et in re mechanica abuntur,' Acta Eruditorum, vol. 3, pp. 161-163, 1686.

[2] I. Newton, Philosophiae Naturalis Principia Mathematica, London, 1687.

[3] A. Einstein, Annalen der Physik, vol. 18, pp. 639-641, 1905.

[4] E. Schrödinger, Physical Review, vol. 28, pp. 1049-1070, 1926.

[5] G. W. Leibniz, "Specimen dynamicum pro admirandis Naturae legibus circa corporum vires et mutuas actiones detegendis, et ad suas causas revocandis," Acta Eruditorum, vol. 4, pp. 145-157, 1695.

[6] Albert Einstein, "Über das relativitätsprinzip und die aus demselben gezogenen folgerungen," Jahrbuch der Radioaktivität und Elektronik, vol. 4, pp. 411-462, 1907.

[7] O. Klein, Zeitschrift für Physik, vol. 37, no. 12, pp. 895-906, 1926.

[8] W. Gordon, Zeitschrift für Physik, vol. 40, pp. 117-133, 1926-1927.

[9] P. A. M. Dirac, "Series A, Containing papers of a mathematical and physical character," in Proc. the Royal Society of London. 1928, pp. 610-624.

[10] I. Barukčić, "Anti Heisenberg-refutation of Heisenberg's uncertainty relation," in Proc. American Institute of Physics Conference, 2011, pp. 322-325.

[11] I. Barukčić, "Anti-Bell-Refutation of Bell's theorem," in Proc. American Institute of Physics Conference, 2012, pp. 354-358.

[12] I. Barukčić, "Anti-Bell-Refutation of Bell's theorem," in Proc. American Institute of Physics Conference, 2012, pp. 354-358.

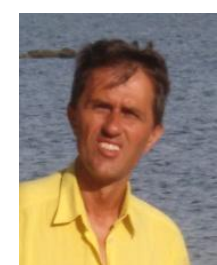

Ilija Barukčić was born October $1^{\text {st }}, 1961$ in Novo Selo (Bosina and Hercegovina, former Yugoslavia). He studies at the University of Hamburg from 1982 to 1989), Germany. He ends of studies with the degree: State exam. His basic field of research is the relationship between cause and effect under conditions of quantum and relativity theory, in biomedical sciences, in philosophy et cetera. Thus far, among his main publications in physics, published by the American Institute of Physics, are the refutation of Heisenberg's uncertainty principle (Ilija Barukčić, "Anti Heisenberg - Refutation Of Heisenberg's Uncertainty Relation," American Institute of Physics - Conference Proceedings, Volume 1327, pp. 322-325, 2011), the refutation of Bell's theorem and the CHSH inequality (Ilija Barukčić, "Anti-Bell - Refutation of Bell's theorem," American Institute of Physics - Conference Proceedings, Volume 1508, pp. 354-358, 2012.). In another publication, he was able to provide the proof that time and the gravitational field are equivalent (Ilija Barukčić, "The Equivalence of Time and Gravitational Field,” Physics Procedia, Volume 22, pp. 56-62, 2011). 